\title{
Ecotoxicologia de biofertilizante bovino e ovino
}

Ecotoxicology of bovine and sheep biofertilizers

Ecotoxicología de biofertilizantes bovinos y ovinos

Gislayne de Araujo Bitencourt Professora Doutora, Universidade Estadual de Mato Grosso do Sul -UEMS, Brasil gislaynebitencourt@gmail.com

Larissa Maria Vaso Agrônoma, Mestranda, Instituto Agronômico de Campinas - IAC, Brasil. larissamvaso@gmail.com

Aurélio Luis Carpinedo Gomez Agrônomo, Mestrando Universidade Estadual de Mato Grosso do Sul -UEMS, Brasil. aurelioluiscg@gmail.com

Thainara Trindade Souza Agrônoma, Universidade Estadual de Mato Grosso do Sul -UEMS, Brasil. thaitrindadesouza@gmail.com

\section{Bruno Sotolane Pradebon} Agrônomo, Universidade Estadual de Mato Grosso do Sul -UEMS, Brasil. brunospradebon@gmail.com

Bruno Ervolino Montanhez Doutorando, Universidade Federal de Mato Grosso do Sul - UFMS, Brasil. brunomontanhez@gmail.com 


\begin{abstract}
RESUMO
Os biofertilizantes com base de dejetos animais, apresentam grande quantidade de nutrientes que permitem sua utilização como adubo. Entretanto, se faz necessária sua caracterização química, pois podem apresentar efeitos de toxicidade para o solo e planta. Objetivou-se com este trabalho avaliar os efeitos da aplicação de dois biofertilizantes na germinação e crescimento inicial de Zea mays L. e Phaseolus vulgaris L. O experimento foi realizado em casa de vegetação, em vasos contendo $3 \mathrm{~kg}$ de solo e os biofertilizantes foram diluídos em água deionizada nas concentrações de: $0,150,300,450$ e $600 \mathrm{~mL}$. L $\mathrm{L}^{-1}$. O delineamento utilizado foi inteiramente casualizado (DIC) em fatorial $2 \times 5$ (biofertilizantes $x$ concentrações) com cinco repetições e duas espécies. As variáveis avaliadas foram: índice de velocidade de germinação, porcentagem de germinação, comprimento de raiz e de parte aérea. Os dados foram submetidos a análise de variância e teste de Tukey a $5 \%$ de probabilidade utilizando o programa SISVAR. Com base nos resultados, os biofertilizantes não apresentaram efeitos tóxicos nas espécies avaliadas. $O$ biofertilizante bovino apresentou resultados superiores ao ovino. A concentração de $300 \mathrm{~mL} . \mathrm{L}^{-1}$ do fertilizante bovino estimulou aumento de massa seca de parte aérea no feijoeiro e no milho promoveu aumento no crescimento e massa seca de parte aérea. Os biofertilizantes apresentaram potencial de reutilização no cultivo dos vegetais sem ocasionar efeitos tóxicos.
\end{abstract}

PALAVRAS-CHAVE: Adubo. Bioensaio. Dejetos Animais.

\begin{abstract}
Biofertilizers based on animal waste, have a large amount of nutrients that allow their use as fertilizer. However, its chemical characterization is necessary, as they may present toxicity effects for the soil and plant. The objective of this work was to evaluate the effects of the application of two biofertilizers on the germination and initial growth of Zea mays L. and Phaseolus vulgaris L. The experiment was carried out in a greenhouse, in pots containing $3 \mathrm{~kg}$ of soil and the biofertilizers were diluted in deionized water in the concentrations of: $0,150,300,450$ and $600 \mathrm{~mL}$. $\mathrm{L}^{-1}$. The design used was completely randomized (DIC) in factorial $2 \times 5$ (biofertilizers $x$ concentrations) with five replications and two species. The variables evaluated were: germination speed index, percentage of germination, root and shoot length. The data were submitted to analysis of variance and Tukey's test at 5\% probability using the SISVAR program. Based on the results, biofertilizers did not present toxic effects on the species evaluated. The bovine biofertilizer showed superior results to the sheep. The concentration of $300 \mathrm{~mL}$. $\mathrm{L}^{-1}$ of the bovine fertilizer stimulated an increase in shoot dry matter in beans and in corn promoted an increase in shoot growth and dry mass. Biofertilizers showed potential for reuse in plants without causing toxic effects.
\end{abstract}

KEYWORDS: Fertilizer. Bioassay. Animal waste.

\title{
RESUMEN
}

Los biofertilizantes basados en desechos animales tienen una gran cantidad de nutrientes que permiten su uso como fertilizante. Sin embargo, su caracterización química es necesaria, ya que pueden presentar efectos de toxicidad para el suelo y la planta. El objetivo de este trabajo fue evaluar los efectos de la aplicación de dos biofertilizantes en la germinación y el crecimiento inicial de Zea mays L. y Phaseolus vulgaris L. El experimento se llevó a cabo en un invernadero, en macetas que contenían $3 \mathrm{~kg}$ de suelo y los biofertilizantes se diluyeron en agua desionizada en las concentraciones de: $0,150,300,450$ y $600 \mathrm{~mL}$. $\mathrm{L}^{-1}$. El diseño utilizado fue completamente al azar (DIC) en factorial $2 \mathrm{x}$ 5 (biofertilizantes $x$ concentraciones) con cinco repeticiones y dos especies. Las variables evaluadas fueron: índice de velocidad de germinación, porcentaje de germinación, longitud de raíz y brote. Los datos fueron sometidos a análisis de varianza y prueba de Tukey con un 5\% de probabilidad utilizando el programa SISVAR. Según los resultados, los biofertilizantes no presentaron efectos tóxicos en las especies evaluadas. El biofertilizante bovino mostró resultados superiores a las ovejas. La concentración de $300 \mathrm{~mL}$. $\mathrm{L}^{-1}$ del fertilizante bovino estimuló un aumento en la materia seca del brote en frijoles y en el maíz promovió un aumento en el crecimiento del brote y la masa seca. Los biofertilizantes mostraron potencial de reutilización en el cultivo de vegetales sin causar efectos tóxicos.

PALABRAS CLAVE: Fertilizante. Bioensayo Desecho animal. 


\section{INTRODUÇÃO}

Os resíduos gerados da produção animal, do ponto de vista econômico e ambiental podem ser utilizados como biofertilizantes. Seu tratamento em biodigestores converte dejetos sem valor comercial em energia e adubo (QUADROS et al. 2010).

$O$ uso dos biofertilizantes na forma de adubo, surge como alternativa para reduzir ou substituir a utilização de insumos sintéticos no solo e nas plantas (SÁ et al., 2015). Segundo Trani et al. (2013), os materiais orgânicos variam conforme a constituição, origem, teor de umidade e processo de estabilização. Estes materiais proporcionam vários benefícios no solo, como a melhoria da estrutura do solo, infiltração de água e aeração. Seu uso estimula a atividade microbiológica e enzimática, promove a liberação de nutrientes que resulta no maior crescimento e nutrição das plantas (OLIVEIRA et al., 2014).

Várias pesquisas relatam o uso benéfico dos biofertilizantes em plantas. Oliveira et al. (2014) cita seu uso na pimenteira. Outros autores mencionam o uso em abóbora (SANTOS et al., 2012); feijão (GALBIATTI et al.; 2011); meloeiro (SANTOS et al., 2014); milho (MATA et al., 2010); tomate (GOMES JÚNIOR et al., 2011).

Embora os biofertilizantes apresentem vários benefícios, os mesmos podem apresentar potencial contaminante. Por esse motivo, a utilização sem restrições depende do conhecimento da composição química (nutrientes e metais pesados) e microbiológica a fim de evitar contaminações (RAMIRES et al., 2019).

Os testes ecotoxicológicos com organismos terrestres são utilizados na detecção dos efeitos nocivos dos resíduos agroindustriais, para conhecer o grau ou o efeito biológico. Para tanto, são realizados com espécies bioindicadoras padronizadas que apresentam pequeno limite de tolerância ecológica a determinadas substâncias químicas, que manifestem alterações fisiológica, morfológica ou comportamental quando expostos a essas substâncias (BARSZCZ et al., 2019; RAMIRES et al., 2019).

A partir dessa premissa, objetivou-se avaliar o efeito de dois biofertilizantes na germinação e crescimento inicial de Phaseolus vulgaris L. e Zea mays L. por meio de teste ecotoxicológico.

\section{MATERIAL E MÉTODOS}

O experimento foi conduzido na Universidade Estadual de Mato Grosso do Sul, Unidade Universitária de Aquidauana, coordenadas $55^{\circ} 67^{\prime}$ Oeste e $20^{\circ} 45^{\prime}$ Sul, de clima tropical subúmido ( $\mathrm{Aw}$ ) com verão chuvoso e inverno seco, temperatura média anual de $26^{\circ} \mathrm{C}$, região ecótono Cerrado - Pantanal.

Os biofertilizantes bovino e ovino foram cedidos pelo Laboratório de Tratamento de Resíduos da mesma unidade. Foram produzidos utilizando biodigestores tipo batelada, com capacidade de 2 litros de substrato em fermentação. Os mesmos, foram abastecidos com água + dejetos contendo aproximadamente $6 \%(\mathrm{~m}: \mathrm{v})$, sólidos totais sendo operado com TRH (tempo de retenção hidráulica) de 90 dias em condições anaeróbias.

Foi aferido o $\mathrm{pH}$ e a condutividade elétrica (CE) dos dois biofertilizantes com o auxílio de um pHmetro e condutivímetro conforme metodologia proposta por Abreu et al. (2009). 
A quantificação de nitrogênio foi realizada de acordo com o método de digestão com $\mathrm{H}_{2} \mathrm{SO}_{4}+$ $\mathrm{H}_{2} \mathrm{O}_{2}$ e destilação de Kjeldahl conforme metodologia descrita por Miyazama et al. (2009) e de fósforo disponível de acordo com o método de Mehlich 1, a leitura foi realizada utilizando um espectrofotômetro no comprimento de onda de $660 \mathrm{~nm}$ de acordo com a metodologia proposta por Silva et al. (2009). A matéria orgânica foi quantificada com base no método de Walkley-Black segundo Embrapa (1997).

Os coliformes totais e termotolerantes foram quantificados por meio do teste de tubo múltiplo conforme Soares e Maia (1999).

Os biofertilizantes foram diluídos em água deionizada nas concentrações: 0 (água deionizada),

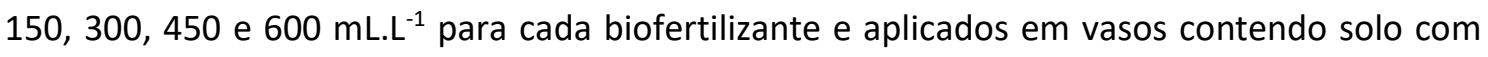
capacidade de $3 \mathrm{~kg}$.

O solo utilizado do tipo Argissolo Vermelho distrófico típico (SCHIAVO, 2010) foi coletado na camada de 0-30 cm, em área de campo, setor de horticultura da UEMS. O mesmo foi seco ao ar livre, seguido de peneiramento manual (com peneira de malha $5 \mathrm{~mm}$ ). Uma subamostra foi caracterizada quanto sua fertilidade pelo laboratório de análises do IAGRO (Tabela 1).

Tabela 1: Atributos químico do solo coletado e utilizado no experimento

\begin{tabular}{|c|c|c|c|c|c|c|c|}
\hline $\begin{array}{c}\mathrm{pH} \\
\mathrm{CaCl}_{2}\end{array}$ & MO & V & $P$ & $\mathrm{Fe}$ & $\mathrm{Mn}$ & $\mathrm{Cu}$ & $\mathrm{Zn}$ \\
\hline$\ldots \ldots \ldots \ldots$ & g. $\mathrm{kg}^{-1}$ & $\%$ & & $\ldots \ldots \ldots \ldots \ldots \ldots$ & g. $\mathrm{dm}^{-3}$ & $\ldots$ & \\
\hline 6 & 22 & 63 & 142,8 & 16,23 & 7,15 & 0,27 & 3,94 \\
\hline CE & K & $\mathrm{Ca}$ & $\mathrm{Mg}$ & $\mathrm{Al}$ & $\mathrm{H}^{+} \mathrm{Al}$ & $S$ & CTC \\
\hline $\mathrm{mS} . \mathrm{cm}^{-1}$ & & $\ldots \ldots \ldots$ & $\ldots \ldots \ldots \ldots \ldots \ldots$ & olc. $\mathrm{dm}^{-}$ & $\ldots \ldots \ldots \ldots \ldots \ldots$ & $\ldots \ldots \ldots \ldots$ & \\
\hline 34,9 & 0,38 & 4 & 0,7 & 0 & 3 & 5,11 & 8,11 \\
\hline
\end{tabular}
bases; $\mathrm{P}=$ fósforo disponível extraído por $\mathrm{Melich}^{-1} ; \mathrm{Fe}=$ ferro disponível; $\mathrm{Mn}=$ manganês; $\mathrm{Cu}=$ cobre; $\mathrm{Zn}=$ zinco; $\mathrm{K}=$ potássio trocável; $\mathrm{Ca}$ = cálcio trocável; $\mathrm{Mg}$ = magnésio trocável; $\mathrm{Al}=$ alumínio; $\mathrm{H}+\mathrm{Al}$ = acidez trocável; $\mathrm{S}=$ soma de bases; $\mathrm{CTC}=$ capacidade de troca catiônica

O experimento foi realizado em condições de casa de vegetação com temperatura média aproximada de $26^{\circ} \mathrm{C}$, a umidade do solo foi mantida em $40 \%$ da capacidade de retenção de água, por meio da pesagem dos vasos e adição de água destilada conforme metodologia descrita na norma ISO 11269-1 (1993).

O teste de germinação e crescimento vegetal foi baseado na norma 11269-2 (2014), foram semeadas 10 sementes de feijão carioca BRS ESTILO e Milho híbrido MG600PW Dow AgroSciences em cada vaso. A porcentagem de germinação (G\%) e o índice de velocidade de germinação (IVG) foi avaliada durante os sete primeiros dias da semeadura com base em Carvalho e Carvalho (2009). Após a emergência de sete plântulas no vaso controle (contendo apenas solo), foi realizado o desbaste de todos os tratamentos, de forma aleatória, totalizando sete plântulas por vaso. 
O delineamento experimental utilizado foi inteiramente casualizado (DIC) em fatorial $2 \times 5$ (dois biofertilizantes e cinco concentrações). Cada vaso correspondeu a uma repetição, totalizando cinco repetições de cada tratamento para cada cultura. As culturas foram avaliadas separadamente.

Após 21 dias do plantio, as plantas foram removidas dos vasos e lavadas em água e, avaliados os seguintes parâmetros: comprimento de parte aérea (CPA) e radicular (CR), massa seca das raízes (MSR) e parte aérea (MSPA).

Para obtenção do comprimento utilizou uma fita métrica e os dados foram obtidos em $\mathrm{cm}$. As raízes e a parte aérea das plantas foram separadas, secas em estufa de aeração forçada a $65^{\circ} \mathrm{C}$ por 72 horas. Posteriormente, foram pesadas em balança analítica para a obtenção da biomassa. Após, foram moídas em moinho para a obtenção de um pó fino para a quantificação de nitrogênio amoniacal e de fósforo disponível (MIYAZAMA et al., 2009; SILVA et al., 2009).

As amostras de solo incorporadas com os biofertilizantes nas diferentes concentrações logo após a instalação do experimento e ao final (21 dias) foram coletadas e secas em estufa de aeração forçada a $65^{\circ} \mathrm{C}$ por 72 horas, foram utilizadas para análise do potencial hidrogênio iônico $(\mathrm{pH})$ em pHmetro, condutividade elétrica (CE) em condutivímetro segundo metodologias de Abreu et al. (2009), nitrogênio amoniacal (MIYAZAMA et al. 2009) e de fósforo disponível (SILVA et al., 2009).

Os dados obtidos foram submetidos a análise de variância (teste $F$ ) e as médias foram comparadas por meio do teste de Tukey $(\mathrm{p}<0,05)$ utilizando o programa estatístico SISVAR (FERREIRA, 2019).

\section{RESULTADOS E DISCUSSÃO}

Os valores referentes ao $\mathrm{pH}$, condutividade elétrica, nitrogênio, fósforo, matéria orgânica e, coliformes totais e termotolerantes dos dois biofertilizantes estão apresentados na tabela 2.

Tabela 2: Caracterização dos parâmetros físico-químico dos dois biofertilizantes.

\begin{tabular}{|c|c|c|c|c|c|c|c|}
\hline \multicolumn{8}{|c|}{$\mathrm{pH}$} \\
\hline & $\mathrm{CaCl}_{2}$ & $\begin{array}{c}\mathrm{Ce} \\
\left(\mathrm{mS}^{\left.-\mathrm{cm}^{-1}\right)}\right.\end{array}$ & $\begin{array}{c}\mathrm{N} \\
\left(\mathrm{g} \cdot \mathrm{kg}^{-1}\right) \\
\end{array}$ & $\begin{array}{c}P \\
\left(\mathrm{mg} \cdot \mathrm{dm}^{-3}\right)\end{array}$ & $\begin{array}{c}\mathrm{MO} \\
\left(\mathrm{g} \cdot \mathrm{kg}^{-1}\right) \\
\end{array}$ & $\begin{array}{l}\text { СТО } \\
(\mathrm{mL}) \\
\end{array}$ & $\begin{array}{l}\text { CTE } \\
(\mathrm{mL}) \\
\end{array}$ \\
\hline Bovino & 8,57 & 6,83 & 22,89 & 175,01 & 28,19 & $2,5.10^{-2}$ & $0,4 \cdot 10^{-2}$ \\
\hline Ovino & 8,10 & 2,60 & 24,08 & 38,54 & 28,19 & $1,5.10^{-2}$ & $0,3 \cdot 10^{-2}$ \\
\hline
\end{tabular}

$\mathrm{pH}$ = potencial hidrogênio iônico; $\mathrm{Ce}=$ condutividade elétrica; $\mathrm{N}=$ nitrogênio; $\mathrm{P}=$ fósforo disponível; $\mathrm{MO}=$ matéria orgânica; CTO = coliformes totais; CTE = coliformes termotolerantes.

Os biofertilizantes apresentaram valores de $\mathrm{pH}$ alcalino, de acordo com a Resolução CONAMA no 430/2011 (BRASIL, 2011) estão de acordo com o intervalo de pH ideal que define como padrão o $\mathrm{pH}$ de 5 a 9 para efluentes de origem biológica.

A condutividade elétrica refere-se a quantidade de sais presentes na solução, os biofertilizantes apresentaram elevada condutividade, sendo $6,83 \mathrm{mS}_{\mathrm{cm}} \mathrm{cm}^{-1}$ no bovino e de $2,60 \mathrm{mS} . \mathrm{cm}^{-1}$ no ovino.

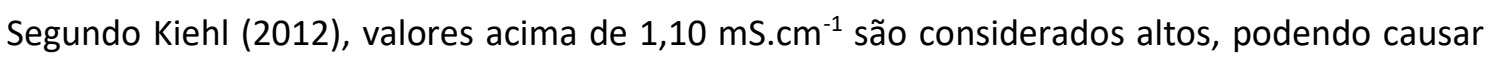


danos nas sementes e interferir na germinalidade, devido à alta concentração de sais ocasionado um estresse salino.

A respeito dos macronutrientes primários, a concentração de nitrogênio foi semelhante nos dois biofertilizantes. Entretanto, a quantidade de fósforo é mais elevada no fertilizante bovino, sendo $78 \%$ maior do que o ovino.

Os biofertilizantes foram enquadrados na classe $A$, de acordo com a resolução CONAMA no 375 , de 29 de agosto de 2006 (BRASIL, 2006). Os valores de coliformes totais e termotolerantes estão dentro do limite máximo permitido pela resolução podendo ser reutilizado na agricultura sem causar danos na fauna e na flora.

Os valores de pH e CE do solo com aplicações dos biofertilizantes estão descritos na Tabela 3.

Tabela 3: Caracterização dos parâmetros físico-químico do solo com aplicação de biofertilizante bovino e ovino nas diferentes concentrações.

\begin{tabular}{ccccc}
\hline & \multicolumn{2}{c}{ Bovino } & \multicolumn{2}{c}{ Ovino } \\
\hline $\mathrm{mL} . \mathrm{L}^{-1}$ & $\mathrm{pH}$ & $\mathrm{CE}\left(\mu \mathrm{S} . \mathrm{cm}^{-1}\right)$ & $\mathrm{pH}$ & $\mathrm{CE}\left(\mu \mathrm{S} . \mathrm{cm}^{-1}\right)$ \\
\hline 0 & 6,72 & 34,9 & 6,72 & 34,9 \\
150 & 6,42 & 40,0 & 6,65 & 40,8 \\
300 & 6,48 & 55,7 & 6,55 & 48,3 \\
450 & 6,57 & 56,7 & 6,52 & 66,0 \\
600 & 6,58 & 85,5 & 6,57 & 72,3 \\
\hline
\end{tabular}

$\mathrm{pH}=$ potencial hidrogênio iônico; $\mathrm{CE}$ = condutividade elétrica do substrato

$\mathrm{O} \mathrm{pH}$ manteve próximo da neutralidade em todas as concentrações nos dois biofertilizantes. A CE aumentou de forma crescente de acordo com o incremento da concentração aplicada, não ultrapassando o valor de $1,0 \mathrm{mS} . \mathrm{cm}^{-1}$. Os parâmetros avaliados nos biofertilizantes foram considerados adequados para o cultivo vegetal (Kiehl, 2012).

Na Tabela 4 estão apresentados o resumo da análise de variância para os parâmetros avaliados obtidas pelo teste $\mathrm{F}$ no experimento com $P$. vulgaris.

Tabela 4: Resumo da análise de variância do índice de velocidade de germinação (IVG), porcentagem de germinação (G\%), comprimento de parte aérea (CPA) e de raiz (CR), massa seca de parte aérea (MSPA) e de raiz (MSR) de feijão (Phaseolus vulgaris L.) nas diferentes concentrações dos dois biofertilizantes.

\begin{tabular}{lccccccc}
\hline FV & GL & IVG & G\% & CPA & CR & MSPA & MSR \\
\hline Bio & 1 & $14,01^{*}$ & $14,56^{*}$ & 0,02 & 0,12 & $1851,2^{*}$ & 1,37 \\
Conc & 4 & 1,07 & 2,08 & 2,25 & 0,95 & 1,83 & 2,44 \\
Bio*Conc & 4 & 1,39 & $3,68^{*}$ & 0,66 & 1,07 & 0,39 & 2,20 \\
Erro & 40 & & & & & &
\end{tabular}

*significativo $(p<0,05)$. FV = Fonte de variação; GL = graus de liberdade; Bio = biofertilizante; Conc = concentração.

Foi verificada diferença significativa $(p<0,05)$ pelo teste de Tukey nas variáveis IVG, G\% e MSPA de feijão nas diferentes concentrações e entre os dois biofertilizantes (Tabela 5). 
Tabela 5: Valores médios estimados para o índice de velocidade de germinação (IVG), porcentagem de germinação (G\%) e massa seca de parte aérea (MSPA) de feijão (Phaseolus vulgaris L.) nas diferentes concentrações dos dois biofertilizantes.

\begin{tabular}{lllllll}
\hline & \multicolumn{2}{c}{ IVG } & \multicolumn{2}{c}{ Germinação (\%) } & \multicolumn{2}{c}{ MSPA } \\
$\mathrm{mL} . \mathrm{L}^{-1}$ & Bovino & Ovino & Bovino & Ovino & Bovino & Ovino \\
\hline 0 & $0,62 \mathrm{aA}$ & $0,61 \mathrm{aA}$ & $82,0 \mathrm{aA}$ & $78,0 \mathrm{aA}$ & $8,81 \mathrm{aA}$ & $3,31 \mathrm{aB}$ \\
150 & $0,59 \mathrm{aA}$ & $0,44 \mathrm{abA}$ & $88,0 \mathrm{aA}$ & $90,0 \mathrm{aA}$ & $8,72 \mathrm{aA}$ & $3,59 \mathrm{aB}$ \\
300 & $0,64 \mathrm{aA}$ & $0,50 \mathrm{abA}$ & $86,0 \mathrm{aA}$ & $98,0 \mathrm{aA}$ & $8,91 \mathrm{aA}$ & $3,49 \mathrm{aB}$ \\
400 & $0,62 \mathrm{aA}$ & $0,39 \mathrm{abB}$ & $78,0 \mathrm{aB}$ & $92,0 \mathrm{aA}$ & $9,31 \mathrm{aA}$ & $3,70 \mathrm{aB}$ \\
600 & $0,65 \mathrm{aA}$ & $0,31 \mathrm{bB}$ & $68,0 \mathrm{aA}$ & $98,0 \mathrm{aA}$ & $9,17 \mathrm{aA}$ & $3,72 \mathrm{aB}$ \\
\hline
\end{tabular}

Médias seguidas de letras iguais, minúsculas nas colunas e maiúsculas nas linhas, não diferem entre si pelo teste de Tukey a $5 \%$ de probabilidade.

O IVG de feijão não apresentou diferença entre as concentrações no biofertilizante bovino. Entretanto, o ovino promoveu redução nas concentrações de 400 e $600 \mathrm{~mL} . \mathrm{L}^{-1}$. Os dados revelam que o biofertilizante ovino aumentou o tempo de germinação das sementes. Fato esse, pode estar relacionado a alta condutividade elétrica verificada nas concentrações citadas. De modo geral, o fertilizante bovino promoveu os melhores resultados com relação ao ovino nessa variável.

A porcentagem de germinação de feijão não alterou nas diferentes concentrações avaliadas. Foi verificada diferença entre os dois biofertilizantes na concentração de $400 \mathrm{~mL} . \mathrm{L}^{-1}$.

A MSPA do feijoeiro não diferiu nas diferentes concentrações de biofertilizantes. Contudo, apresentou diferença significativa entre os dois biofertilizantes. Nesse caso, o fertilizante bovino promoveu os maiores valores de MSPA. O biofertilizante bovino apresenta em sua composição elevados teores de fósforo comparado ao ovino, sugerindo o aumento na produção de biomassa.

Resultados diferentes foram obtidos com Z. mays. Na Tabela 6 estão apresentados o resumo da análise de variância para os parâmetros avaliados obtidas pelo teste $F$. As variáveis $C P A, C R$, MSPA e MSR de milho apresentaram diferenças significativas $(p<0,05)$ pelo teste de Tukey (Tabela 7).

Tabela 6: Resumo da análise de variância do índice de velocidade de germinação (IVG), porcentagem de germinação (G\%), comprimento de parte aérea (CPA) e de raiz (CR), massa seca de parte aérea (MSPA) e de raiz (MSR) de milho (Zea mays L.) nas diferentes concentrações dos dois biofertilizantes.

\begin{tabular}{lccccccc}
\hline FV & GL & IVG & G\% & CPA & CR & MSPA & MSR \\
\hline Bio & 1 & 0,250 & 0,071 & 2,46 & $6,69^{*}$ & 2,78 & 1,63 \\
Conc & 4 & 1,24 & 0,56 & $8,41^{*}$ & $12,51^{*}$ & $3,88^{*}$ & $6,59^{*}$ \\
Bio*Conc & 4 & 0,28 & 0,39 & 1,69 & 0,89 & 1,0 & 1,41 \\
Erro & 40 & & & & & & \\
\hline
\end{tabular}

*significativo $(p<0,05)$. FV = Fonte de variação; GL = graus de liberdade; Bio = biofertilizante; Conc = concentração. 
Tabela 7: Valores médios estimados para o índice de velocidade de germinação (IVG), porcentagem de germinação

(G\%) e massa seca de parte aérea (MSPA) de milho (Zea mays L.) nas diferentes concentrações dos dois biofertilizantes.

\begin{tabular}{lcccc}
\hline \multicolumn{5}{c}{ CPA } \\
\hline $\mathrm{mL} . \mathrm{L}^{-1}$ & Bovino & Ovino & Bovino & Ovino \\
\hline 0 & $51,62 \mathrm{bA}$ & $51,62 \mathrm{bA}$ & $42,92 \mathrm{aA}$ & $42,95 \mathrm{aA}$ \\
150 & $57,12 \mathrm{abA}$ & $57,59 \mathrm{abA}$ & $38,62 \mathrm{abA}$ & $36,42 \mathrm{abA}$ \\
300 & $61,15 \mathrm{aA}$ & $54,55 \mathrm{abB}$ & $35,60 \mathrm{abA}$ & $29,15 \mathrm{bB}$ \\
400 & $60,59 \mathrm{aA}$ & $60,82 \mathrm{aA}$ & $35,74 \mathrm{abA}$ & $31,54 \mathrm{bA}$ \\
600 & $58,74 \mathrm{aA}$ & $56,61 \mathrm{abA}$ & $33,25 \mathrm{bA}$ & $31,16 \mathrm{bA}$ \\
\hline \multicolumn{5}{c}{ MSPA } \\
\hline $\mathrm{mL} . \mathrm{L}^{-1}$ & Bovino & Ovino & Bovino & Ovino \\
\hline 0 & $1,81 \mathrm{bA}$ & $1,81 \mathrm{aA}$ & $0,70 \mathrm{aA}$ & $0,70 \mathrm{aA}$ \\
150 & $2,23 \mathrm{abA}$ & $2,09 \mathrm{aA}$ & $0,70 \mathrm{aA}$ & $0,78 \mathrm{aA}$ \\
300 & $2,36 \mathrm{aA}$ & $1,91 \mathrm{aB}$ & $0,64 \mathrm{aA}$ & $0,42 \mathrm{bB}$ \\
400 & $2,29 \mathrm{abA}$ & $2,31 \mathrm{aA}$ & $0,58 \mathrm{aA}$ & $0,56 \mathrm{abA}$ \\
600 & $2,11 \mathrm{abA}$ & $1,99 \mathrm{aA}$ & $0,50 \mathrm{aA}$ & $0,40 \mathrm{bA}$ \\
\hline
\end{tabular}

Médias seguidas de letras iguais, minúsculas nas colunas e maiúsculas nas linhas, não diferem entre si pelo teste de Tukey a $5 \%$ de probabilidade.

Os valores referentes ao CPA de milho, todas as concentrações de ambos os biofertilizantes promoveram crescimento superiores ao controle. Entretanto, na concentração de $300 \mathrm{~mL}$. L-1 foi obtida a maior média no biofertilizante bovino. Resultados diferentes foram verificados na variável $C R$, que apresentou a maior média no controle. Ou seja, as concentrações dos biofertilizantes não estimularam o crescimento das raízes.

A respeito da MSPA de milho, resultados superiores foram obtidos com a aplicação do biofertilizante bovino. A concentração de $300 \mathrm{~mL}$. L-1 promoveu o maior incremento de biomassa. $O$ biofertilizante ovino não apresentou diferença significativa entre as concentrações. Em relação a MSR em milho, o biofertilizante bovino não promoveu diferenças entre as concentrações. Inversamente, o fertilizante ovino, promoveu aumento de biomassa na concentração de $150 \mathrm{~mL}$. L $\mathrm{L}^{-1}$.

Segundo Lima et al. (2011), a adição de material orgânico, promove um arranque inicial do crescimento da parte aérea das plantas. A adição de biofertilizante não interferiu no crescimento de raiz e parte aérea do feijoeiro. Contudo, o biofertilizante bovino proporcionou incremento de massa seca de parte aérea em todas as concentrações aplicadas. Na cultura de milho, o biofertilizante bovino promoveu aumento nas variáveis CPA e MSPA na concentração de $300 \mathrm{~mL}$. $\mathrm{L}^{-1}$.

Os resultados corroboram com as observações de alguns autores que utilizaram biofertilizantes de origem animal. Pinheiro et al. (2019) cita o uso benéfico do esterco bovino e caprino no cultivo de feijão caupi. Sousa et al. (2013), verificou aumento linear na altura de planta, diâmetro do caule e número de ramos em pinhão manso pelas concentrações de biofertilizante bovino. Figueiredo et al. (2011), utilizando esterco ovino verificou o aumento na produção de alface e da atividade microbiana do solo. Castilhos et al. (2007) verificaram que o vermicomposto a base 
de esterco ovino promoveu maior matéria seca em plantas de alface quando comparado com outros resíduos como esterco bovino, borra de café e erva mate.

Com base nos resultados obtidos por Martins et al. (2015), o esterco bovino isolado ou associado ao biofertilizante pode substituir o adubo mineral na produção de feijão comum. Contudo, ressalta que o biofertilizante não devem ser empregados isoladamente.

De acordo com Galbiatti et al. (2010) a melhor produtividade de feijoeiro foi obtida nos tratamentos que receberam o efluente de biodigestor à base de esterco bovino, podendo ser equivalente a adubação química.

De acordo com Guazzelli et al. (2012), para verificar o efeito positivo do biofertilizante, o mesmo deve ser aplicado na dose adequada, em pequenas quantidades e de forma parcelada. Cancellier et al. (2011) ressaltam que, para que a adubação orgânica tenha efeitos significativos na produtividade, se faz necessária a aplicação por vários anos, maximizado seus efeitos a longo prazo, resultando na melhoria na fertilidade do solo e consequentemente no desenvolvimento dos vegetais.

Outro aspecto a ser considerado, é com relação a espécie avaliada e qual o estágio de desenvolvimento em que o estresse é aplicado. Segundo Dias e Blanco (2010), ressalta que o crescimento inicial é bastante sensível ao excesso de sais e que a resposta a esse estresse pode variar conforme a espécie, genótipo, estágio fenológico e o tempo de exposição.

O experimento evidenciou que são necessários mais estudos sobre a aplicação dos biofertilizantes. Sugerimos um cultivo por maior período e parcelamento das dosagens, aumentando o tempo de ação do biofertilizante nos vegetais, para que os nutrientes sejam acumulados no solo e disponibilizado para as plantas.

\section{CONCLUSÕES}

1. Os biofertilizantes têm potencial de utilização, não foram verificados efeitos tóxicos nas espécies avaliadas.

2. O biofertilizante bovino apresentou resultados superiores ao ovino.

3. A concentração de $300 \mathrm{~mL} . \mathrm{L}^{-1}$ estimulou aumento das variáveis avaliadas.

4. No feijoeiro, o biofertilizante bovino promoveu incremento de massa seca de parte aérea.

5. Foram observados aumento de crescimento e massa seca de parte aérea de milho quando cultivado com biofertilizante bovino.

\section{REFERÊNCIAS BIBLIOGRÁFICAS}

ABREU, M. F. de.; ABREU JUNIOR, C. H.; SILVA, F.C. da.; SANTOS, G. C. G.; ANDRADE, J. C.; GOMES, T. F.; COSCIONE, A. R.; ANDRADE, C. A. Análises químicas de fertilizantes orgânicos (urbanos). In: SILVA, F.C. DA. Manual de análises químicas de solos, plantas e fertilizantes do solo. 2.ed. Brasília, DF: Embrapa Informação Tecnológica, 2009. (p.401-405).

BARSZCZ, L.B.; BELLATO, F.C.; BENASSI, R.F.; MATHEUS, D.R. Avaliação ecotoxicológica de efluentes tratados por alagados construídos. Engenharia Sanitaria e Ambiental, v. 24, n.6, p. 1147-1156, 2019.

https://dx.doi.org/10.1590/s1413-41522019184120 
BRASIL. Ministério do Meio Ambiente. Conselho Nacional do Meio Ambiente - CONAMA. Resolução no 375, de 29 de agosto de 2006. Define critérios e procedimentos, para o uso agrícola de lodos de esgoto gerados em estações de tratamento de esgoto sanitário e seus produtos derivados, e dá outras providências. Diário Oficial da República Federativa do Brasil, Brasília, DF, 30 de agosto de 2006.

http://www2.mma.gov.br/port/conama/res/res06/res37506.pdf

BRASIL. Ministério do Meio Ambiente. Resolução CONAMA № 430, DE 13 DE MAIO DE 2011, Dispõe sobre as condições e padrões de lançamento de efluentes, Complementa e altera a Resolução no 357, de 17 de março de 2005, do Conselho Nacional do Meio Ambiente-CONAMA. Diário Oficial da União, Brasília, 16 de Maio. 2011. http://www.adasa.df.gov.br/images/stories/anexos/8Legislacao/Res CONAMA/Resolucao CONAMA 430 2011.pd $\underline{f}$

CANCELLIER, L.L.; AFFÉRRI, F.S.; ADORIAN, G.C.; RODRIGUES, H.V.M.; MELO, A.V.; PIRES, L.P.M.; CANCALLIER, E.L. Adubação orgânica na linha de semeadura no desenvolvimento e produtividade do milho. Revista Semina, v.32, n.2, abr./ jun. p.527 540, 2011. http://dx.doi.org/10.5433/1679-0359.2011v32n2p527.

CARVALHO, B.D.; CARVALHO, R.I. Qualidade fisiológica de sementes de guanxuma em influência do envelhecimento acelerado e da luz. Acta Scientiarum Agronomy, v. 31, n. 3, p. 489-494, 2009.

CASTILHOS, D.D.; SOUZA, LM.; MORSELLI, T.B.G.A.; CASTILHOS, R.M.V. 2007. Alterações químicas no solo e produção de alface decorrentes da adição de vermicompostos. Magistra, v.19:, p.143-149, 2007.

DIAS, N. S.; BLANCO, F. F. Efeitos dos sais no solo e na planta. In.: Gehyi, H. R.; Dias, N. S.; Lacerda, C. F. Manejo da salinidade na agricultura: Estudos básicos e aplicados. Fortaleza-CE, INCTSal., p.133- 144, 2010.

EMBRAPA. Empresa Brasileira de Pesquisa Agropecuária. Centro Nacional de Pesquisa de Solos. Manual de métodos de análise de Solo. 2 ed. rev. atual. Rio de Janeiro, 1997. 212 p. (EMBRAPA-CNPS. Documentos, 1).

FERREIRA, D.F. SISVAR: a computer analysis system to fixed effects split plot type designs. Revista brasileira de biometria, v.37, n.4, p.529-535, 2019.

FIGUEIREDO, C.C.de.; RAMOS, M.L.G.; MCMANUS, C.M.; MENEZES, A.M.de. Mineralização de esterco de ovinos e sua influência na produção de alface. Horticultura Brasileira, v.30, p. 175-179, 2012.

GALBIATTI, J.A.; SILVA, F.G. da.; CLAUDENIR F.; FRANCO, C.F.; CARAMELO, A.D. Desenvolvimento do feijoeiro sob o uso de biofertilizante e adubação mineral. Engenharia Agrícola, Jaboticabal, v.31, n.1, p.167-177, 2011.

GOMES JÚNIOR, J.; SILVA, A.J.N. da.; SILVA, L.L.M.; SOUZA, F.T.de.; SILVA, J.R. da.; Crescimento e produtividade de tomateiros do grupo cereja em função da aplicação de biofertilizante líquido e fungo micorrízico arbuscular. Revista Brasileira de Ciências Agrárias, v.6, n.4, p.627-633, 2011. DOI:10.5039/agraria.v6i4a1362

GUAZELLI, M.J.B.; RUPP, L.C.D.; VENTURINI, L. Biofertilizantes. Programa de Fortalecimento da Viticultura Familiar da Serra Gaucha. SL: Grafisul. 13p. 2012. (MDA/IBRAVIN. Publicação Técnica, 1).

INTERNATIONAL ORGANIZATION FOR STANDARDIZATION. ISO 11269-1: Soil quality - Determination of the effects of pollutants on soil flora - Part 1: Method for the measurement of inhibition of root growth. Geneva, 1993.

INTERNATIONAL ORGANIZATION FOR STANDARDIZATION.ISO 1269:2. Qualidade do solo - determinação dos efeitos de poluentes na flora terrestre. 2. ed. Rio de Janeiro, 2014. 23 p. 
KIEHL, R.J. Manual de compostagem: maturação e qualidade do composto. 6. ed. Piracicaba: O autor, 2012. 171 p.

LIMA, R.L.S.; SEVERINO, L.S.; SOFIATTI, V.; GHEYI, H.R.; CARVALHO JÚNIOR, G.S.; ARRIEL, N.H.C. Crescimento e nutrição de mudas de pinhão manso em substrato contendo composto de lixo orgânico. Revista Caatinga, v.24, n.3, p.167-172, 2011.

MARTINS, J.D.L.; MOURA, M.F.de.; OLIVEIRA, J.P.F.de.; OLIVEIRA, M.de.; GALINDO, C.A.F. Esterco bovino, biofertilizante, inoculante e combinações no desempenho produtivo do feijão comum. Revista Agro@mbiente, v.9, n.4, p.369-376. 2015. http://dx.doi.org/10.18227/1982-8470ragro.v9i4.2583

MATA, J. F.; SILVA, J. C. da.; RIBEIRO, J. F.; AFFÉRRI, F. S.; VIEIRA, L. M. Produção de milho híbrido sob doses de esterco bovino. Pesquisa Aplicada \& Agrotecnologia, v.3, n.3, p.125-134, 2010.

MIYAZAWA, M.; PAVAN, M.A.; MURAOKA, T.; CARMO, C.A.F.S.; MELO, W.J. Análise química de tecido vegetal. In: In: SILVA, F.C. DA. Manual de análises químicas de solos, plantas e fertilizantes do solo. 2.ed. Brasília, DF: Embrapa Informação Tecnológica, 2009. (p. 198-204).

OLIVEIRA, J.R. de; GOMES, R.L.F.; ARAÚJO, A.S.F.; MARINI, F.S.; LOPES, J.B.; ARAÚJO, R.M. Estado nutricional e produção da pimenteira com uso de biofertilizantes líquidos. Revista Brasileira de Engenharia Agrícola e Ambiental, v.18, n.12, p.1241-1246, 2014. https://doi.org/10.1590/1807-1929/agriambi.v18n12p1241-1246

PINHEIRO, R. A.; SANTOS, D. R.; CABRAL, M. J. dos S.; SILVA, R. de A.; DE BARROS, R. P. de. Atributos biológicos do feijão caupi (Vigna unguiculata (L.) Walp., fabaceae) cultivado em vasos com diferentes fontes de adubação orgânica. Revista Ambientale, v.11, n.3, p.15-25, 2019. https://doi.org/10.34032/ambientale.v11i3.161

QUADROS, D. G; OLIVER, A. P. M.; REGIS, U.; VALLADARES, R.; SOUZA, P. H. F.; FERREIRA, E. J.; Biodigestão anaeróbia de dejetos de caprinos e ovinos em reator contínuo de PVC flexível. Revista Brasileira de Engenharia Agrícola e Ambiental, v. 14, n. 3, p. 326- 332, 2010. DOI: https://doi.org/10.1590/S1415-43662010000300014.

RAMIRES, M.F.; SOUZA, E.L. de; FONTANIVE, D.E.; BIANCHETTO, R.; KRYNSKI, A.M.; CEZIMBRA, J.C.G.; ANTONIOLLI, Z.I. Ecotoxicology of Pig Slaughterhouse Waste Using Lactuca sativa L., Raphanus sativus L., and Oryza sativa L.. Revista Brasileira de Ciência do Solo, v. 43, e0180119, 2019. https://doi.org/10.1590/18069657rbcs20180119

SÁ, F.V. da. S.; MESQUITA, E.F. de.; BERTINO, A.M.P.; COSTA, J.D.; ARAÚJO, J.L. Influência do gesso e biofertilizante nos atributos químicos de um solo salino-sódico e no crescimento inicial do girassol. Irriga, Botucatu, v. 20, n. 1, p. 46-59, 2015. DOI: https://doi.org/10.15809/irriga.2015v20n1p46

SANTOS, M.R. dos.;, SEDIYAMA, M.A.N; MOREIRA, M.A.; MEGGUER, C.A.; VIDIGAL, S.M. Rendimento, qualidade e absorção de nutrientes pelos frutos de abóbora em função de doses de biofertilizante. Horticultura Brasileira, v.30, n.1, p.160-167, 2012. https://doi.org/10.1590/S0102-05362012000100027

SANTOS, A.P.G.; VIANA, T.V.A.; SOUSA, G.G, GOMES-DO-Ó, L.M, AZEVEDO, B.M.; SANTOS, A.M. Produtividade e qualidade de frutos do meloeiro em função de tipos e doses de biofertilizantes. Horticultura Brasileira, v.32, n.4, p. 409-416, 2014. https://doi.org/10.1590/S0102-053620140000400007

SCHIAVO, J. A.; PEREIRA, M.G.; MIRANDA, L.P.M.DE.; DIAS NETO, A. H. D.; FONTANA, A. Caracterização e classificação de solos desenvolvidos de arenitos da formação Aquidauana - MS. Revista Brasileira de Ciência do Solo, n.34, p. 881-889, 2010.

SILVA, F.C. da.; ABREU, M.F. de. PEREZ, D.V.; EIRA, P. A. da.; ABREU, C.A. de.; RAIJ, B.V.; GIANELLLO, C.; COELHO, A.M.; QUAGGIO, J.A.; TEDESCO, M.J.; SILVA, C.A.; BARRETO, W.O. Métodos de análises químicas para a avaliação 
da fertilidade do solo. In: SILVA, F.C. DA. Manual de análises químicas de solos, plantas e fertilizantes do solo. 2.ed. Brasília, DF: Embrapa Informação Tecnológica, 2009. (p.130-134).

SOARES, J.B.; MAIA, A.C.F. Água: microbiologia e tratamento. Fortaleza: UFC, 1999. 206p.

SOUSA, G.G. de.; VIANA, T. V. de. A.; BRAGA, E.S.; AZEVEDO, B.M.de.; MARINHO, A.B.; BORGES, F.R.M. Fertirrigação com biofertilizante bovino: Efeitos no crescimento, trocas gasosas e na produtividade do pinhão-manso. Agrária Revista Brasileira de Ciências Agrárias, v.8, n.3, p.503-509, 2013.

TRANI, P.E.; TERRA, M.M.; TECCHIO, M.A.; TEIXEIRA, L.A.J.; HANASIRO, J. Adubação orgânica de hortaliças e frutíferas. Campinas: Instituto Agronômico de Campinas; 2013. 